\title{
Estructura y fundamentación Ex ante para la definición de Modelos de Gestión Organizacional en las PyMEs*
}

\section{Structure and foundation Ex ante for the definition of models of organizational management in SMEs}

\section{Struktur und fundament Ex ante für die definition von Modellen der Organisations management in KMU}

Jahir A. Gutiérrez O. ${ }^{1}$, José M. Gutiérrez L." ${ }^{2}$ Elimeleth Asprilla M. ${ }^{3}$

\section{Resumen}

$\mathrm{Fl}$ artículo analiza el grado de correspondencia entre los modelos o teorías de gestión organizacional a la hora de contextualizarlos en el mundo empresarial de las PyMEs. El comportamiento, especialmente particular de estas estructuras, impide la generalización de algún tipo de modelo a la hora de explicar su desempeño. La evidencia empírica destaca, que las PyMEs deben explicarse desde la base misma de su origen, y frente a ello, cualquier asomo explicativo termina siendo insuficiente. En primer lugar, se revisa el alcance que puede tener la fundamentación de modelos de gestión organizacional en las PyMEs. Segundo, la capacidad de absorción que tienen estas a la hora de considerarlos en sus estructuras, y finalmente, las condiciones que se requieren para incorporarlos. En esencia, la modelación organizacional en la PyME es un reto para la investigación.

Palabras claves: comportamiento de la empresa; economía de la empresa; organización industrial; organización y comportamiento de la empresa.

\begin{abstract}
The article analyses the degree of correspondence between models or theories of organizational management when it comes to contextualize them in the business world for SMEs. The behavior, especially particular of these structures, prevents the generalization of some kind of model in explaining his performance. Empirical evidence highlights, that SMEs must be explained from the same base of its origin, and in response, any explanatory hint ends up being insufficient. Firstly, the scope that can be the Foundation of organizational management models in SMEs is reviewed. Second, the absorptive capacity that have these when considering them in their structures, and finally, the conditions required to incorporate them. In essence, organizational modeling in SMEs is a challenge for research.
\end{abstract}

Keywords: behavior of the company; economy of the enterprise; industrial organization; Organization and performance of the company.

\section{Zusammenfassung}

Der Beitrag analysiert den Grad der Übereinstimmung zwischen den Modellen oder Theorien der Organisationsmanagement, wenn es ihnen in der Geschäftswelt für KMU kontextualisieren kommt. Das Verhalten, vor allem insbesondere dieser Strukturen, verhindert, dass die Verallgemeinerung von irgendeiner Art von Modell zu erklären seine Leistung. Empirische Belege unterstreicht, dass KMU müssen von der gleichen Basis seiner Herkunft erklärt werden, und in der Antwort, endet jede erläuternde Hinweis unzureichend. Erstens, der Umfang, die der Gründung der Organisationsmanagementmodelle in KMU werden kann, ist überprüft. Zweitens, die Aufnahmefähigkeit, die diese bei der Prüfung sie in ihren Strukturen, und schließlich haben, benötigt die Bedingungen, um sie $\mathrm{zu}$ integrieren. Im Wesentlichen Organisationsmodellierung in $\mathrm{KMU}$ ist eme Herausforderung für die Forschung.

* Este articulo es producto del proyecto de investigación: Modelo de Gestión de PyMEs, realizado por el Grupo de Investigación RFD- GFA, Categoría B en Colciencias y el Grupo de Investigación Observatorio Público, ambos de la Institución Universitaria Tecnológico de Antioquia. Aprobado por la Dirección de Investigaciones de la Institución Universitaria Tecnológico de Antioquia, entre el periodo de Octubre 2011 a Octubre de 2012

1 - Magíster en Desarrollo.Universidad Pontificia Bolivariana. Medellín.Colombia. jagutier@tdea.edu.co

2 - Especialista en Mercadeo. Universidad EAFIT. Medellín. Colombia. josemagutierrez@gmail.com

3 - Economista. Universidad Simón Bolívar. Barranquilla.Colombia. eliasmo@tdea.edu.co 


\section{Introducción}

La puesta de modelos organizacionales destinados a formalizar las actividades empresariales, representa la posibilidad de obtener elementos de trabajo sobre estas. No obstante, la modelación empresarial en cualquier versión, es sólo un marco de evidencia empírica. En primer lugar, la modelación debe sustentar completamente los elementos que la representan. Segundo, el aval y refrendación empresarial del mismo es fundamental, además que, la generalización o réplica del modelo es crucial, a la hora de inferir el comportamiento de modelo o ejemplo.

El escenario en que se plantean los modelos organizacionales en el marco de las empresas PyME, no han evolucionado del carácter apriorístico y de evidencia empírica. Entre otras, el carácter de empresas comúnmente informales o de naturaleza particular en diferentes casos, ha provocado que los resultados en materia de modelación se tomen de igual forma. Por ende, los modelos obtenidos a través de estas u otras formas empresariales, es sólo un abrebocas inicial del análisis que implica rastrear los resultados que la modelación ofrece finalmente.

La revisión del modelo debe contar con el análisis apriorístico o propositivo de las empresas consultadas inicialmente en el marco del proyecto o por la evidencia empírica. Si el modelo fue obtenido a través de ellas, resulta totalmente improcedente, respaldar lo generado en el modelo por cuenta de la revisión teórica u otras modelaciones. En este sentido, la modelación en la PyME, responde a escenarios estrictamente particulares, en los que el objeto de estudio pasa de alternar su disposición como evidencia, a participar en la consecución del modelo.

El proceso de modelación en cualquiera de sus versiones, diseño, modelo o prototipo en la PyME u otra forma empresarial, debe superar el connato de evidencia empírica o de resultado final. La modelación debe hacer un recorrido superior al trazado en los objetivos del proyecto que le da origen. Por ende, la trazabilidad, entendida como la trayectoria que recorre el modelo para afianzarse como tal, es un recorrido poco mostrado en las investigaciones, dejando a los resultados obtenidos en solo aproximaciones de lo que implica estudiar la PyME.

La estructura temática del artículo obedece a los siguientes temas de contenido: En primer lugar, sobre los fundamentos para la elaboración e ilustración de modelos de gestión organizacional en la PyME. Segundo, los límites y restricciones que se tienen para acoger modelos empresariales en las PyME, y tercero, la estructura que requiere la modelación en la PyME, para que efectivamente sea reconocido dicho ejercicio. Es la empresa, quien debe validar dichos modelos, aún en estudios de casos, so pena de quedar solo en evidencias.
1. Fundamento para la Elaboración e Ilustración de Modelos de Gestión Organizacional en las PyME

La modelación de la gestión organizacional de la PyME debe alternarse claramente los intereses que puede generar dicho propósito con los intereses de ellas. Si, el proyecto que le da origen solo plantea preguntas a priori, no consultadas con la PyME, la premisa de elaborar cualquier modelo termina presentando rezagos y por ende viciada. Si bien, es importante reconocer el trasfondo por parte de dichos acercamientos, la validación hecha por el empresario o desde la empresa, es sumamente vital para el éxito del proyecto. Plantea Ayuzabet (2002:25):

Dado que el objeto de estudio de la Teoría Organizacional (TO) son las organizaciones modernas y dado que éstas aparecen a mediados del siglo XIX -y por tanto, pueden ser consideradas como hijas de la modernidad industrial, la racionalización organizacional y la racionalidad económica-, la TO puede ser considerada en primera instancia como la respuesta teórica a los problemas enfrentados por la gran empresa moderna, es decir, problemas de productividad, estructura, estrategia, mercado, competencia, desarrollo tecnológico, etcétera.

El sólo acople del modelo, requiere un trabajo más amplio fuera de las intenciones con que se convalidan al aplicarlos. Los preceptos con que se elaboran modelos en cualquier escenario, exige una especie de repliegue sobre el que éstos son fundamentados. A su vez, el interés que generan a la hora de tener asiento a manera de ejemplo en cualquier ámbito, no es suficiente para que sean postulados directamente como modelos susceptibles de aplicación, si éstos no contienen para dicha réplica, sustentación para avalar su apuesta y formalización. Sostiene Bernal y otros (2010:42-43):

Los resultados de este estudio destacan que las organizaciones consideradas de tamaño mediano tienden a tener un mejor grado de gestión del conocimiento que sus homólogas de mayor o menor tamaño. Este hecho es un resultado interesante con relación a posteriores estudios sobre el tema.

En cuanto a los análisis, estudios e investigaciones del orden empresarial, ha ganado terreno la posibilidad de extender la aplicación de modelos, sin formalización plena de manera recurrente. El permanente recurso de utilizarlos para evidenciar diferentes aspectos, exige igualmente una corroboración elemental de los aspectos que hacen posible al mismo. El acervo de elementos que requiere en primera instancia la constitución de los modelos, no solo está sujeto a las condiciones, parámetros, supuestos y variables sobre los que son plasmados. Expone Blanco (2010:341):

"El enfoque no es un análisis estático de la empresa, tomando una fotografía de la misma, sino el análisis del conjunto de elementos teóricos y de 
evidencia empírica del aprendizaje, evolución e integración de las empresas".

La modelación corre el riesgo básicamente de presentar problemas de adaptabilidad o reciprocidad con respecto al junto de empresas estudiadas. En este sentido, el modelo debe tener capacidad de flexibilidad para especificar las razones por las cuales no se ubica por completo en la empresa. Es relevante a la hora de formalizar cualquier ejercicio de adaptación y adopción de modelos, más que la apropiación directa del mismo, la apuesta de las condiciones sobre las cuales éste pasaría de la modelación al acoplamiento explicativo en una actividad. Analiza Livacic (2009:190):

"Por ello, junto con valorar como orientaciones sus aspectos positivos, a la hora del análisis hay que seguir más de cerca a autores clásicos, de probada vigencia a través del tiempo. Y recordar que las Organizaciones son el reflejo de nuestra vida como sociedad".

La bondad de los modelos, radica en la posibilidad de orientar nuevas posibilidades, tanto para quienes los catalogan de fundamentales a la hora de realizar estudios 0 , imprescindibles para quienes recurren a la categorización, como mecanismo para entender el comportamiento de las empresas. Ésta es la razón que impulsa a exigir y postular un marco de trabajo más propio a la hora de escenificar modelos. La contundencia de los modelos, no radica en su acoplamiento o repetición, sobre el cual solo genera estado del arte. Considera Estrada (2010:176):

Diseñar planes, construir modelos, estructurar posibles esquemas de acción son condiciones naturales de la racionalidad gerencial. De lo que se desprende una amplia idea sobre la facultad directiva en la organización.

Sin embargo, mientras más alcance tenga la consolidación de un modelo o de propuestas similares, el trabajo se vuelve más aprehensivo, irrumpiendo con cualquier connato de inferencia que puedan ofrecer las encuestas o entrevistas. El entrar de la mano con las instituciones que proveen datos e información o que son cercanas a la realidad que se pretende analizar, es un punto de referencia para convalidar el esfuerzo, normalmente académico y de investigación, cuando se trata de proveer elementos de trabajo a un ente $u$ objeto de trabajo. Indican Herrera y Osorio (2006:8687):

El éxito de la gestión no radica solamente en el modelo mismo, sino que existen factores como la cultura organizacional y algunas condiciones de negociación asociadas que podrían impedir el resultado positivo de la gestión. Para terminar, es importante aclarar que el modelo por sí sólo no funciona si no se realiza una apropiación de parte del personal encargado, y se tiene plena confianza en los resultados que él arroje.
La estilización y extensión de los modelos se hace más que necesaria a la hora de asumir que uno de ellos puede ser acogido o escenificado en una realidad concreta, de lo contrario sin premeditación, éste termina siendo una copia lineal. La certificación hace parte de las cualidades con las que debe contar los ejercicios convalidados bajo la raigambre de modelos. Así, que la sola mediación entre datos obtenidos y revisión de las características con las que fueron adquiridas no es suficiente, al contrario, va en dirección opuesta al origen que les dio vigencia. Ver Tabla 1.

Tabla 1. Evolución de modelos organizacionales

\begin{tabular}{|l|l|l|l|}
\hline Espacio histórico & $1900-1980$ & $1981-1990$ & $1991-\imath ?$ \\
\hline Etapa histórica & Estandarización & Orientación al cliente & Innovación \\
\hline $\begin{array}{l}\text { Estructura } \\
\text { organizacional }\end{array}$ & $\begin{array}{l}\text { Jerarquizada } \\
\text { (funcional, divisional, } \\
\text { matricial) }\end{array}$ & $\begin{array}{l}\text { Adhocracia } \\
\text { Horizontal, Red }\end{array}$ & Celular \\
\hline Activo clave & Bienes de capital & Información & Conocimiento \\
\hline Gerente clave & $\begin{array}{l}\text { Gerente de } \\
\text { operaciones }\end{array}$ & $\begin{array}{l}\text { Gerente de } \\
\text { informática }\end{array}$ & $\begin{array}{l}\text { Gerente de } \\
\text { conocimiento } \\
\text { (Chief Knowledge } \\
\text { officer CKO) }\end{array}$ \\
\hline $\begin{array}{l}\text { Competencias } \\
\text { clave }\end{array}$ & $\begin{array}{l}\text { Especiaiización } \\
\text { y segmentación }\end{array}$ & $\begin{array}{l}\text { Flexibilidad } \\
\text { y capacidad } \\
\text { de respuesta }\end{array}$ & $\begin{array}{l}\text { Diseño de } \\
\text { la creatividad }\end{array}$ \\
\hline
\end{tabular}

Fuente: Adaptado a partir de Miles Raymond - Charles Snow et al. Organizing in the knowledge age: Anticipating the celular form. Academy of management executlve. 1997. Vol. 11 No. 4.

En: Rivas T (1998:15).

El poder construir modelos propios sobre los que pueda analizarse el comportamiento empresarial y de cara a ello entregar elementos para la aplicación en ellas mismas, sirve de incentivo para que los trabajos no terminen afincados en mecanismos que pueden desorientar la trascendencia de lo que se busca. En este sentido, la ilustración propia surgida en las pesquisas o revisiones hechas por quienes elaboran el modelo, así como la inferencia que aparece con la evaluación lineal de los datos, desluce cualquier trabajo que apunte a la definición de modelo. Reitera Tovar (1998:44):

Los reportes internacionales de la aplicación de estos modelos son escasos en calidad y número por lo cual, las investigaciones y los informes siguen esta misma tendencia. Sin embargo, su conocimiento y difusión propiciarán una versatilidad en el diseño de nuevas formas de organización.

Si sobre éste no recae el contraste, los ejercicios de prueba - error, ajuste o incluso consideración bajo diversas metodologías, lo que se recoge finalmente no es ni siquiera las bases del modelo que se pretende diseñar o elaborar sino escasamente sus conclusiones. Es a eso, a lo que estamos expuestos cuando bajo la ausencia de filtros son filtrados modelos aplicados. Es la preocupación constructiva la que obliga a repensar el afán por orientar cualquier intervención en las 
empresas o de otros cortes, bajo el precepto de modelo. Contextualiza Lloréns (1996:131):

Actualmente, la mayoría de los investigadores de las áreas relacionadas con la dirección y la administración de empresas están de acuerdo en la necesidad de desarrollar teorías contrastables empíricamente, y que éstas sean lo suficientemente complejas como para dar un conocimiento más profundo y exacto de la realidad.

En ningún caso del orden cultural, educativo, empresarial, productivo y social, puede haber espacio para dilatar la necesidad de procurar evidencias $y$ ejemplos, sobre los cuales contrastar la contundencia y fuerza con que se pretende allanar la aplicación o presencia de algún tipo de modelo. Es como, una especie de rectificación permanente, a la hora de establecer que conforme a los criterios con que ha sido elaborado el modelo fuente, éste puede ser considerado como ejemplo a escala local, a la hora de convocar a la entrega de los resultados. Anota Riquelme y otros (2009:43):

"Esta gestión del conocimiento permite explicar los factores o elementos de apoyo que facilitan los procesos de interacción de la organización con el entorno y aquellos que nacen en forma interna2.

La generación de ensayos prueba - error o el empuje para producir diferentes ejercicios con los cuales pueda establecerse la resiliencia del modelo, hace parte del compromiso que se tiene a la hora de convocar a la aplicación, proposición o revisión de cualquier modelo. No basta con la existencia y réplica de este en otros escenarios; al respecto, es necesario llevar el caso en cuestión a la revisión a través de diferentes metodologías para con ellas, mostrar en cada uno de los espacios comparados, las advertencias, problemáticas o resultados del modelo. Considera Louffat (2004:103):

El inicio del siglo XXI nos permite observar una serie de cambios en el campo organizacional, en su identidad estratégica, estructural y operacional, los cuales han modificado profundamente las bases de las ciencias administrativas, poniendo en jaque sus paradigmas tradicionales.

Las empresas tienen claro el camino a seguir o por lo menos así lo hacen saber, por ello, recurren a los estudios e investigaciones para palpar las oportunidades que pueden tener en distintos campos. Preguntarles sobre competitividad, diseño, gestión, mercados, precios y resultados, suena redundante en actividades que requieren certeza en sus actividades y exploración de oportunidades, y no una especie de exploración que resulta contraproducente frente a sus requerimientos. La empresa en Colombia bajo cualquiera de sus denominaciones, propiamente no ha mostrado interés por ser enmarcada en cualquier figuración. Ver Tabla 2.
Tabla 2.

Canales de Transferencia modelos organizacionales

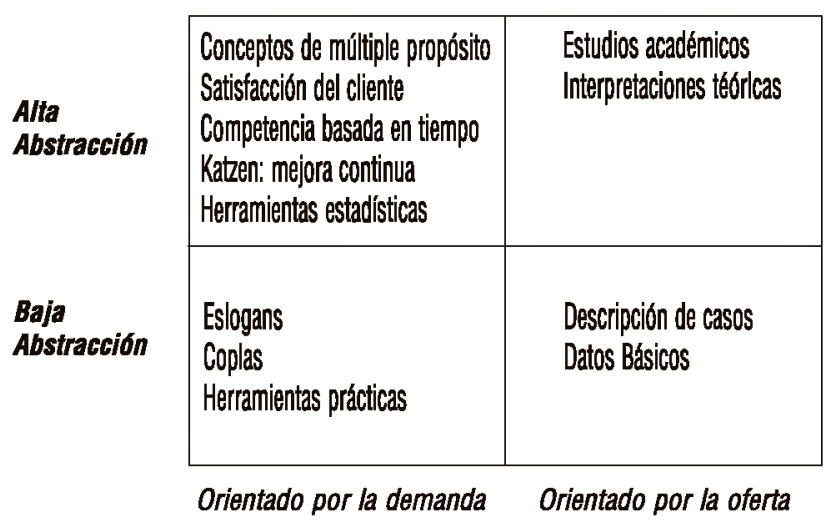

Fuente: Lilirank (1994:977)

\section{En: Montaño (2000:253).}

El papel que cumplen las agremiaciones a la hora de orientar los trabajos que pretenden formalizar directa o indirectamente algún tipo de comportamiento empresarial se hace más que necesario. Si bien, los estudios de casos son importantes en cualquier etapa de la revisión básica de los estudios, no representan ejercicios formales con los cuales pueda estimarse bajo interés algún tipo de postura empresarial. Por ende, es necesario que se haga un trabajo constante de manera interna y externa, para que los análisis sean constatados. Explica Montaño (2000: 252):

La transferencia implica entonces la elaboración de un modelo que considera sólo algunos aspectos -de los denominados formales- como el diseño estructural, políticas de personal, capacitación, tecnología, pero se desentiende de los demás. De esta forma se genera una especie de modelo universal, que da la apariencia de poder operarse en cualquier tipo de organización sin importar la región geográfica. Círculos de calidad, just in time, kaizen, kanban, mejoramiento continuo, son ejemplos claros de esta tendencia.

La sola revisión de estudios o aplicación de casos, solo constituye la enmarcación de trabajos sobre hipótesis nulas, bajo con o sin datos e información se considera que las respuestas están más que expeditas. Por ende, si se quiere hablar de la presencia de un modelo éste debe pasar por una amplia revisión interinstitucional. El superar la interacción a través de encuestas a través de la formalización de un trabajo gremial e institucional, sobre el cual fundamentar la recreación de cualquier intervención empresarial, es el primer paso. Cuestiona Pérez (2007:74):

Y las preguntas que nacen de esta situación son: ¿Es posible sin una gestión profesional de la estructura organizacional, del ser humano y de la cultura que genera su interrelación en las empresas, generar espacios de creatividad e innovación? ¿Es posible 
que una empresa se modernice al margen de sus trabajadores? ¿Es posible que una empresa sea competitiva si no genera espacios de crecimiento para su gente, que es la generadora directa del conocimiento?

Seguidamente, el trabajo con los empresarios, se ejecuta de manera directa a escala de equipo consultivo organizacional y, de paso, en las instituciones en las que emergen dichos estudios termina por ampararse la posibilidad de acoger la postulación y el trabajo de modelos en cualquier campo como el escenario de trabajo natural. De ahí que, la frecuencia con que se aborden estos ejercicios representa validez. La consideración de distintos estamentos acerca de la contundencia o fortaleza de los modelos constituye solo contrario a la salvedad un primer paso.

2. Límites y Restricciones de la Convalidación y Sustentación de Modelos de Gestión Organizacional en las PyMEs

La recurrencia a diversas metodologías para convalidar los asuntos vinculados a trabajos de campo o definición de comportamientos en cualquier contexto, no pueden pasar inadvertidas. La simplificación que de ella puede hacerse a través de la formulación matemática, la evaluación estadística, la medición econométrica, el análisis experimental, de teoría de juegos, teoría de caos o análisis de fractales hacen parte de los fundamentos que para pertinencia de los estudios e investigaciones, deben sortear los fundamentos y resultados de dichos ejercicios. Establece De la Vara, Anes, Sánchez (2004:4):

Para modelar una organización en primer lugar se deben mantener entrevistas con empleados de las distintas unidades organizacionales de la empresa o con empleados que desempeñen actividades asociadas a distintos roles dentro de la organización. Por otro lado, también es conveniente estudiar toda la documentación disponible relacionada tanto con la actividad de la empresa como con sus políticas de negocio. Los modelos generados en esta fase son: diagrama de contexto, modelo de roles, metas estratégicas $u$ organizacionales, modelos de procesos (con recursos), y asignación de metas a procesos.

Bajo dicha dirección, el poder evidenciar y justificar los elementos matemáticos para iniciar la etapa de consideración de parámetros es crucial para la validación. El fundamento matemático contribuye a perfilar las ecuaciones y formulaciones sobre las cuales se extiende de manera básica las racionalidades sobre las cuales se pretende apoyar inicialmente el ejercicio. De ellas, la derivación del comportamiento de cada uno de los parámetros que se constituyen en las empresas, sirven para establecer los niveles de correspondencia que tienen individualmente y entre sí. Plantea Cathalifaud (2008:91):

Las organizaciones se identifican con la capacidad para movilizar, integrar y orientar actividades hacia el cumplimiento de fines, cuyos resultados se observan por su efecto transformador y de agregación de valor. Es en este sentido que las organizaciones tienen incorporada la posibilidad de comunicarse con sus entornos, preferentemente, mediante sus prestaciones de servicios con otras organizaciones.

El balance que puede arrojar las diferentes formas de interpretación de los parámetros definidos, sirve de marco para cimentar las bondades de alcance y aplicación que pueden tener los elementos definidos. La estructura exponencial, lineal y/o logarítmica con la que se fuerza para mostrar el comportamiento de las ecuaciones constituidas al amparo del estudio en cuestión, hace parte de las pruebas y de la capacidad de resistencia que tienen la formulación, escenario sobre el cual debe abordarse bajo un tamiz ampliamente crítico y propositivo. Analiza Arraut (2008: 195):

Son varios los autores que han recomendado que los estudios de casos que deseen profundizar en el cambio organizacional se lleven a cabo con una presencia continua del investigador en la empresa que es objeto de estudio, destacando la ídoneidad de que éste pueda dedicar tiempo al trabajo de campo dentro de la empresa, incorporándose a la misma como un trabajador más y observando los hechos como testigo directo. Sin embargo, muy pocas empresas permiten lo anterior y facilitan el acceso al investigador.

$\mathrm{Al}$ establecer el rango de ajustes, objeciones y salvedades en las que puede recaer la evaluación vía paramétrica de los datos, considerada a través de la sola prueba nominal de análisis de datos abiertos, es un comienzo necesario para ordenar la trayectoria de la información que se obtiene o para orientar los lineamientos sobre los cuales se aspira a conseguir una información que en muchos de los casos se considera estimada, o en el mejor de ellos, cuando el interés radica en analizar y evidenciar hechos que no son propiamente claros. Reafirma Arraut (2008:195):

Como no constituye una opción factible dentro de la investigación la incorporación del investigador a las empresas, con el fin de disminuir los posibles inconvenientes que de ello se deriven se utilizaron diferentes técnicas de recogida de datos. Estas circunstancias llevan, en la mayoría de los estudios de casos, a utilizar una combinación de técnicas tales como las entrevistas, los cuestionarios, la revisión documental y la colaboración de expertos en el sector.

La secuencia derivada del ejercicio de fundamentación matemática al superar por lo menos la etapa de indicadores o elementos con los cuales puede trabajarse, pasa al escenario de la medición que corre por cuenta de la estadística, que conforme a la presencia de datos en convergencia con la formulación predispuesta sirve 
de base para analizar la cercanía o dispersión tanto de los datos como de las variables que los acompañan en cada etapa considerada. Los resultados en este proceso sirven para denotar la depuración de los datos como de las variables. Sustenta Betancourt (2003:209):

Ante tales rasgos, se ha intentado construir un modelo o iniciar un estilo de acción tendiente a que a partir de la administración y como parte de su esencia (la interdisciplinariedad) se configuren una serie de relaciones que contribuyan al objetivo primordial de conocer esencias, al conocimiento y comprensión de realidades humano-organizacionales y administrativas, respondiendo así a cuestionamientos fundamentales (qué, por qué, cómo y cuándo las organizaciones).

El poder mostrar las perspectivas que cada caso arroja a medida que se trabaja con datos y las variables que en principio, además de formuladas terminan siendo quienes convalidan la suerte de los datos, permite establecer, que la irrigación sobre el comportamiento individual y transversal de las variables representa el producto del trabajo que inicialmente se desarrolló a la hora de presentar las formulaciones iniciales en materia de ecuaciones y, como éstas al paso del forzamiento funcional, terminan por desempeñar un papel trascendental. Argumenta Gil (2007:51):

"Se evidencia que la teoría de las empresas ha ofrecido distintos modelos y soluciones para enfrentar los cambios del entorno y promover la mejora continua de las empresas. Se han enfocado desde diferentes visiones de la realidad empresarial y por ello su impacto ha sido heterogéneo".

La perspectiva que al respecto ofrece la sustentación estadística para poder llegar a este tipo de aseveraciones, corre por cuenta, de los elementos que terminan por considerar en análisis de corto, mediano y largo plazo de las variables en cuestión, aspecto en el que prima además de la buena o excelente consecución de datos, la posibilidad de que el estudio no termine por acusar la bondad de estimadores por encima del análisis. La especificación de cada componente de las variables gira en consonancia con la capacidad explicativa que éstas presentan. Ejemplifica Yanes (2001:131):

En todas ellas también subyace, en mayor o menor medida, el problema de la intervención del investigador que puede introducir sesgo en la información obtenida, con lo que se evoluciona desde técnicas en que la respuesta del sujeto estaba totalmente condicionada por los conceptos iniciales presentados por el investigador hacia técnicas en que la influencia es la mínima posible.

El orden estadístico orienta hacia la posibilidad de medir el comportamiento de cada una de las variables, en tanto factores explicativos, como de correlación, dispersión u outliers, sobre los que se aprecia que deben tener un comportamiento que de cierta manera extiende toda la fundamentación de variables nulas, sobre las que se espera la ubicación del contenido analizado bajo los preceptos que permitieron explicarlos directamente desde el contenido matemático y un marco de resiliencia sobre los cuales se considera que estos terminen comportándose. Argumenta Urgal (2007:25-26):

Es preciso comentar algunas limitaciones que tratarán de solventarse en futuros trabajos de investigación. En primer lugar, el análisis estadístico realizado sólo permite confirmar asociaciones, pero no relaciones causales. En segundo lugar, la influencia en el rendimiento empresarial de los programas de inversión en infraestructura de producción puede ser negativa o neutral en el corto plazo, pero positiva en el largo plazo. En tercer lugar, este trabajo se limita al estudio de las decisiones de producción de carácter infraestructural. Sería preciso analizar las cuestiones planteadas en este trabajo en otros ámbitos sectoriales y territoriales para extender las conclusiones obtenidas.

Si bien, la fundamentación matemática y evaluación estadística prima a la hora de promulgar la consistencia de un modelo, el análisis econométrico permite considerar diferentes fenómenos a la hora de apreciar la posibilidad de que el modelo además de explicativo sea predictivo. El propósito de la vinculación de la econometría es precisamente, tomar las valoraciones y resultados del modelo y ponerlos al servicio de la predicción. La estipulación de un modelo por cuenta de las directrices econométricas ubica la discusión a un nivel mucho más explicativo. Estiman Etxebarria y Sánchez (2008:165):

Si a este planteamiento se le añade la consideración de la empresa como un sistema racional (orientado hacia objetivos especificados) aparece una categoría de aportaciones, especialmente relevante en el ámbito de la teoría de la organización, como es la de los modelos de sistemas racionales abiertos. Dentro de esta categoría, se incluyen aportaciones como la de la escuela del comportamiento o de los sistemas sociales y la teoría de sistemas.

El alcance que puede tener la predicción estriba en la estipulación de diferentes escenarios o comportamientos que conforme a los datos, índice, indicadores e información obtenida conducen al analista a presentar diferentes simulaciones o evaluaciones con el mismo modelo en diferentes momentos. Incluso, permite trabajar con distintos datos de distintas temporalidades para establecer la sostenibilidad del modelo en el tiempo o si éste se comporta de manera aleatoria o carece de explicación analítica conforme se agregan más variables o se eliminan. Depuran Dini y Stumpo (2011:6):

La baja cobertura de las políticas de apoyo a las pymes (en todas las áreas, no solo en el fomento a la innovación) en América Latina en parte se explica 
porque los programas están diseñados pensando en una cierta categoría de empresas, las más dinámicas, que están en condiciones de definir de forma clara sus demandas y pueden aprovechar de manera eficiente los instrumentos disponibles. Sin embargo, entre tanto, hay un número relevante de empresas que no están en estas condiciones.

La evaluación econométrica, permite abonar a los datos, la parametrización y la cuantificación simulada de los mismos, las interpretaciones que puede tener el modelo a saber. Incluso, el origen de los datos pasa la cuenta cuando estos no tienen fundamentos estadísticos o paramétricos sobre los cuales apoyar cualquier desenlace inesperado con respecto a los resultados que se esperan obtener o que por lo menos representen las expectativas sobre las cuales se decidió intervenir en estipular un comportamiento particular como el descrito en el modelo a saber. Agrega Orayen (2008:61):

Por experiencia de trabajo en empresas pymes hemos verificado que la implementación de modelos de planificación estratégica desarrollados para grandes organizaciones y empresas, tienen una compleja aplicabilidad -por la especificidad de las prácticas- en empresas pymes.

El relevar elementos comunes entre la econometría y la estadística en la mayoría de las casos arrojan aspectos que impiden ver más allá de la necesidad de ajustar, hacer pruebas o corregir algunos de los comportamientos de las variables. Este principio de estilización, no produce más que un aló de tranquilidad para quien bajo los lineamientos ortodoxos considera la inconveniencia de mostrar un modelo que para los entendidos presenta errores que están vigentes en cualquiera de las etapas de la simulación o que hacen parte de los ejercicios de aplicación. Indica Naclerio (2010: 25-26):

Se intenta retomar la problemática teórica percibida al analizar el concepto de trama productiva y, además, se considera que esta imprecisión está muy lejos de ser propiedad exclusiva de este concepto en particular. Por el contrario, es posible apreciar que la producción intelectual sobre desarrollo regional y grupos de empresas muestra, en líneas generales, una combinación de excesiva conceptualización con una -en algunos casos importante dosis de vaguedad y ambivalencia en sus definiciones, lo que resulta un caldo de cultivo más que propicio para que se produzca este tipo de imprecisiones.

La manera como se obtienen los datos, los elementos con los cuales se abona la información y los registros tras los que se elaboran las ecuaciones son igual de relevantes a la aplicación de las bases estadísticas que deben guiar los criterios bajo los que se pretende diseñar tras de ciertas pruebas básicas o estimaciones iniciales el posible alcance y tendencia que podría traer el modelo a seguir. Es hora, de permitir que la conjuga- ción matemática - estadística respalde cualquier propuesta que enmarque los estudios sobre determinantes, evaluación y modelación vistos desde el análisis econométrico o el data panel. Plantean Cobacho \& Bosch (2001:3):

Los modelos de datos panel se clasifican en dinámicos o estáticos según incluyan o no en sus ecuaciones variables pertenecientes a diferentes periodos temporales. En la especificación estática, el modelo de regresión de un solo factor supone que el error aleatorio se descompone en $\varepsilon_{i t}=\alpha_{i}+v$ it, donde cada i es el efecto individual (inobservado) de cada unidad de sección cruzada, invariante en el tiempo. El modelo a seguir es el siguiente:

$$
y_{i t}=\alpha_{i}+X_{i t} \beta+v \text { it }
$$

La presencia del efecto fijo en esta ecuación hace que una estimación $\beta$ por MCO no sea consistente. Los métodos que se utilizan para solventar ese problema son la estimación de efectos fijos (EF) o entre grupos (withn groups) y la estimación de efectos aleatorios de EF puede llevarse a cabo transformando el modelo en otro en términos de las medias de grupo:

$$
y_{i t-} y_{i}=\left(X_{i t}-X_{i}\right)^{\prime} \beta++\left(v_{i t}-v_{i}\right)
$$

El preguntar de manera directa a las empresas sobre la realidad de los elementos que sirven de apoyo para el análisis en cuestión con respecto a los modelos, ahorra un trabajo arduo en cuanto a la validación de los instrumentos con los que en no pocos casos se intenta construir información para hacer de ella cuna de diversas inferencias que no clarifican realmente hasta que tanto la consideración de distintas respuestas puede servir para cimentar categóricamente la posible presencia de modelo. Sin embargo, la corroboración hace parte de sus evidencias. Caracteriza Cardona y Gutiérrez (2010:128):

Las pymes se preocupan más por mantenerse dentro del entorno de competitividad que se ha dado gracias a los procesos de globalización, que por pertenecer simplemente a un sector de producción mínimo en el que las opciones de cambio y de sostenimiento no se den a largo plazo.

Es el tránsito natural que deben recibir los elementos que representan la estructura del modelo, que de manera contraproducente termina maleado al criterio y la necesidad de quien está el frente de la digitalización, evaluación, medición o del que realiza las pruebas de ajuste para que el trabajo tenga algún acierto o represente los intereses indicados. La vinculación del sujeto de estudio en la observancia del recorrido que hace el dato y la información en sus distintas acepciones e ilustraciones sirve de articulador y de derivación de los aspectos que explican eficazmente los elementos perseguidos como los que definitivamente no. Sintetiza Mora (2006:108): 
"El uso de los datos de panel va de la mano de los nuevos y difíciles problemas que deberán resolverse particularmente en los modelos no lineales".

Sin la fuente de la información o de quién se desprende los datos que constituyen la fundamentación del modelo, éste no tiene ningún tipo de acercamiento con respecto a la trayectoria que recorre la información desde diferentes connotaciones, se cometen diversos errores, entre tanto, el de corregir información que plenamente es sesgada, que no tiene suficientes criterios explicativos para interpretarlos o considerarlos desde diversas estimaciones y peor aún, se llega a plantear que dadas las condiciones de ellos es necesario ajustarlos o linealizarlos. Arguyen Aguirre y otros (2009:173):

La mayoría de investigaciones de carácter empírico publicadas sugieren que el rendimiento económico empresarial está asociado positivamente con la calidad de la gestión. Sin embargo, de estos estudios no se podían extraer conclusiones concluyentes y definitivas sobre la relación existente entre estas dos dimensiones, especialmente en contextos próximos al nuestro: empresas mayoritariamente de tamaño pequeño y no necesariamente inmersas en programas de calidad.

El poder trabajar de manera directa con quienes sirvieron de base para la obtención de los datos como de la ilustración de diversas conductas que en no pocos casos terminan cuantificadas o medidas, hace parte de los factores de correspondencia y reciprocidad que debe tener cualquier modelo cuando se trata de apreciar como tal y, que a la postre de su maduración, termina por ser puente de explicaciones. Es por ello, que la búsqueda de la cualificación del modelo se hace una razón impostergable a la hora de llevar el ejercicio a difusión o publicación.

\section{Estructura y Elementos para la Difusión de Modelos de Gestión Organizacional en las PyMEs}

Ajustar modelos para la comprensión de los datos y la información es el punto de partida que debe lograr cualquier pretensión de elaborar la estructura de los modelos. Sin embargo, los ajustes están expuestos a la exactitud, convergencia o dispersión de los datos a saber. Es decir, que gran parte del trabajo no consiste propiamente en la utilización del dato, sino de la resistencia que estos tienen para ser contemplados como tal en cada prueba de ajuste, de lo contrario no podría llamarse, bajo el propósito de denominarlo modelo en las áreas empresariales. Señalan Estrada y Dutrénit (2007:292):

Si bien es cierto que no existe un esfuerzo sistemático por desarrollar la gestión, nuestra evidencia empírica permite identificar diversos conjuntos de prácticas intensivas en know how técnico, donde se deposita, comparte y transfiere conocimiento en un contexto muy específico. Tal como se planteaba en nuestro objetivo, estas prácticas expresan diferentes recursos intangibles que operacionalizamos como diversos componentes del capital intelectual.

En contradicción a la posibilidad de elaborar y afincar la estructura del modelo solo con base a interpretaciones extraídas de formulaciones planteadas a través de encuestas u otra forma de información que no tenga fundamentación transversal en la actividad empresarial que es consultada; sin más validación que las aseveraciones o la comparación plena. La disposición para establecer modelos aplicados al comportamiento empresarial conduce a observar de manera directa los alcances obtenidos en el proceso de consolidación de algún modelo en particular. Agrega Peña y Bastidas (2004:58):

"En este sentido, la optimización de la gestión, a través de métodos logísticos, se constituye en un mecanismo cuyo objetivo primordial es optimizar toda la cadena de abastecimiento, desde la misma materia prima hasta la comercialización final".

En este sentido, el análisis sobre modelos empresariales, ha caído en la casuística, mecanismo de origen empírico, pero que no refrenda la trascendencia de las observancias obtenidas a través de la mencionada metodología, cuyas bondades radica precisamente en la revisión sobre los diversos campos en los cuales puede ser replicado el objeto de estudio o la aplicación del modelo a saber. El análisis epistemológico acerca de la postulación de modelos en diversos ejercicios empresariales, es más que necesaria para definir si estos cumplen dicho designio. Destaca Padilla (2008:12):

Frente a ese hecho existe también la necesidad de revisar la parte que le corresponde desarrollar por sí mismas a las PyMEs: sus capacidades de absorción de conocimientos tecnológicos. Habría que agregar que para que esto último pueda ocurrir las PyMEs no pueden solas, como lo demuestran diversas experiencias nacionales (de países del sureste asiático). Las PyMEs necesitan de un marco institucional adecuado y de una política industrial decidida a apoyarlas y fortalecerlas.

El debilitamiento del concepto proviene de la insolvencia analítica y explicativa a la hora de fundamentarlo racionalmente. Se requiere de una formulación más amplia a la hora de proponer consultorías, estudios o investigaciones, cuyo talente sea la revisión o la implementación de un modelo, puesto que la inexistencia de factores explicativos resta validez a los mismos y más aún cuando la información obtenida es asumida como dato, parámetro que no cumple los criterios básicos para ello, a la hora de sustentar los elementos sobre los que deben apoyarse. Aprecia Fúquene y otros (2009:106):

Las herramientas cuantitativas se han trabajado ampliamente como pronóstico, empleándose en diversas áreas, en las cuales han servido para 
obtener mejores decisiones, disminuyendo la incertidumbre al pensar a futuro. Sin embargo, queda claro que esta herramienta no pretende dar una única decisión y que implique ser la correcta; su aporte se evidencia al centrarse en datos $y$ métodos determinísticos que generan menor incertidumbre y mayor aceptación en el ámbito ingenieril.

En este sentido, la ilustración que se hace por diferentes vías para conocer el quehacer de las empresas o formular a partir de ella modelos de comportamiento, entre otros epígrafes, termina siendo vulnerable ante la capacidad de contraste, evaluación y validación propia de ejercicios relacionados. La referencia que se tiene respecto al acercamiento que realizan quienes se interesan en el estudio e investigación de las diferentes actividades empresariales, dista de condiciones fiables para establecer marcos de comportamiento sobre éstas. Aseveran Calderón y Naranjo (2007:166):

Los sistemas culturales y socio estructurales de la organización están fuertemente relacionados. En una organización viable, si el sistema cultural y las características formales de la organización (sus metas, estrategias estructuras y sistemas de gerencia) se han desarrollado de manera concomitante y coherente, la cultura y la estructura estarán relacionadas estrechamente y se apoyarán mutuamente (Allaire y Firsirotu, 1992).

La intensidad hacia la consideración de posibles estructuras de modelos, raya con respecto a las dimensiones sobre las cuales puede determinarse la presencia de los mismos, y para ello, se requiere basta validación y ejercicios permanentes de comprobación. $\mathrm{El}$ análisis empresarial incorporado en distintos frentes de aplicación, supera la irrigación de las preguntas amparadas en la compilación de una serie de preguntas que no alcanzan a establecer con propiedad el carácter y propósito sobre el cual ha sido extendido el análisis a saber. Ver tabla 3.

Tabla 3. Preguntas a PyMEs sobre Modelos de Gestión

\section{Preguntas}

1. ¿Qué entiende se entiende por gestión empresarial?

2. ¿Posee algún tipo de gestión identificado en algún proceso de la empresa?

3. ¿En qué área o proceso le ha traído él mayor impacto?:

4. ¿Se guía "usted" por alguna línea de pensamiento en materia de gestión o tiene presente ideas o de algún empresario que refrende su pensamiento?

5. ¿Conoce algún modelo de gestión empresarial?

6. ¿Pretende en su empresa aplicar o emplear un modelo de gestión en particular?

7. ¿En que periodo de tiempo pretende la empresa implementar o aplicar el modelo de gestión?

8. ¿Por qué y para qué área o sub-área específica de su empresa lo aplicaría o implementaría?

Fuente: Elaboración propia

Es importante que se atienda en forma de cuadrante, los elementos desde los cuales se considera las PyMEs, que en muchos de los casos obedece a relaciones de intercambio simultáneas. El mercado y los precios, la relación entre el capital y el trabajo, los beneficios y costos, y las eficiencias que puedan ser obtenidas con cada proceso productivo cuentan de manera reciproca en la PyME. Frente a ello, las aseveraciones corren por cuenta de quien tiene claro el proceso productivo o la evaluación de los factores de producción con los cuales se obtiene el producto. A su vez, es relevante que las preguntas a la par con las respuestas tiendan a fortalecer las condiciones de gestión que se presentan en las PyMEs. Ver Figura 1.

Figura 1. Modelo de gestión por segmentos

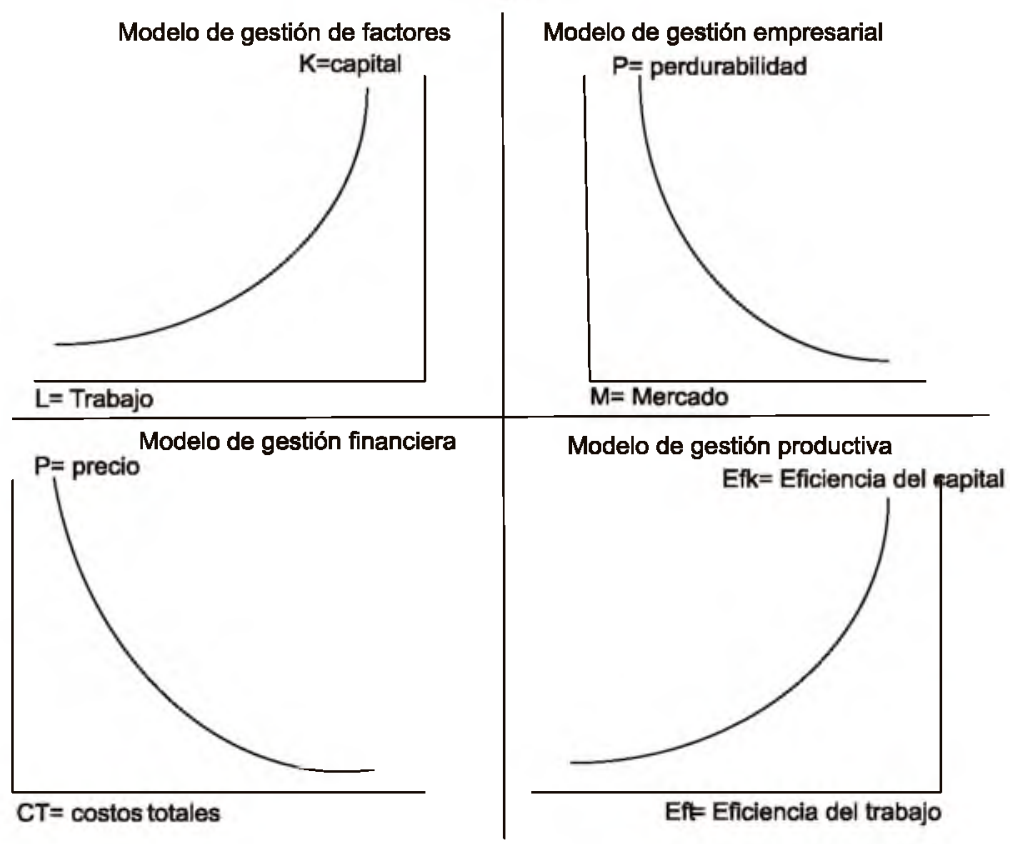


En dichos segmentos se pretende establecer en cada componente que representa para los intereses de las PyMEs la posibilidad de revisar conjuntamente los elementos que de manera reciproca comportan la gestión que para ellas es importante. Es decir, que cualquier práctica que intente definir los lazos de gestión en las PyMEs, requiere primero orientar el interés de éstas a la ahora de apreciar de un área en particular con respecto a la necesidad de facultarla o promoverla como receptáculo de gestión ensimisma y para toda la actividad productiva empresarial. Así que, cada segmento identificado debe estimar la capacidad de generar elementos que le permitan orientar estimaciones de gestión, en cuanto a la importancia y orden que tienen para cada empresa consultada o vinculada a la investigación.

\section{Conclusiones}

El acercamiento a la definición de modelos de gestión en cualquier caso para las PyMEs, no permite abocarse a ellas de manera densa y extensa, puesto que cada área de trabajo y campo de producción de ellas, exige una revisión en términos generales de lo que puede refrendar para dicho propósito el fundamento de modelo de gestión. Es decir, que la realización de trabajos bajo este contexto se alcanza en la medida en que además de identificar áreas en las que puedan incubarse dichos propósitos, estás terminen por explicar el curso de las demás.

La posibilidad de que a través de estos ejercicios pueda ordenarse alguna estructura sobre la cual perfilar el carácter de gestión que podrían tener las PyMEs en cualquier renglón de la actividad, está más sujeto a la directriz gerencial y empresarial de las mismas, que terminan siendo reducibles, mientras en las empresas no se afiancen componentes distintos a los de mercado y producción, dejando la gestión a meras prácticas empresas que no alcanzan el componente de modelo y mucho menos de un orden que conduzca a la gerencia empresarial.

Es importante reconocer que la incubación de los modelos y la forma de hacer las cosas en las PyMEs están sujetas a ellas; al identificar el modelo de gestión al que apuntan o estiman a través de evidencias y resultados. De otro lado, el pretender averiguar en esencia dichos modelos, en cuanto a la revisión general al interior de estas o de forma generalizada, pormenoriza la investigación. El vínculo entre los modelos de gestión con los resultados que puedan tener las PyMEs con su incorporación a la par con la metodología data panel, representa la posibilidad de evidenciar el aliciente que generan los modelos.

\section{Referencias Bibliográficas}

Aguirre G, M. S., Albizu G, E. A., Abando J, C., Forcada S., F. J., Landeta $\mathrm{R}$, J. (2009). Análisis de la calidad en la gestión de las PyMEs de la CAPV y de su incidencia en los resultados económicos. UE. Universidad del País Vasco. Instituto de Economía Aplicada a la Empresa. 1-225.
Arraut C., L. C. (2008). La innovación de tipo organizacional en las empresas manufactureras de Cartagena de indias. Universidad de Medellín, Semestre Económico. 11(22): 185-203.

Ayuzabet De la Rosa A., A (2002). Teoría de la Organización y Nuevo Institucionalismo en el Análisis Organizacional. UAM. Administración y Organizaciones. 15-44.

Bernal T., C. A., Turriago H. Á., Sierra A., H.D. (2010). Aproximación a la medición de la gestión del conocimiento empresarial. ADMINISTER. Universidad EAFTT.(16): 30-49.

Betancourt Z., G. (2003). De la Historia Empresa a la Historia Organizacional. Universidad Nacional de Colombia. INNOVAR. (22): 199-210.

Blanco M., A. C. (2010). Nuevos análisis para la dinámica empresarial: empresa, innovación y desarrollo. Reseñas y debates. Cuadernos de Economía, 29(53): 341-348.

Cabrera, A. y Rincón, M. (2001): "La gestión del conocimiento: creando competitividad en la nueva economía". Información Comercial Española. Revista de economía, $n^{\circ} 791$ (Abril-Mayo), pp. 71-91.

Cathalifaud M., A. (2008). Las Organizaciones desde la Teoría de los Sistemas Sociopoéticos. Cinta Moebio. (32): 90-108. www.moebio.uchile.cl/32/arnold.html

Calderón H G y Naranjo V J C (2007). Perfil cultural de las empresas innovadoras. Un estudio de caso en empresas Metalmecánicas. Cuad. Adm. Bogotá (Colombia), 20 (34): 161-189.

Cardona M., y Gutiérrez O., J. A. (2010). Elementos en el fortalecimiento de los mundos de producción de las Pymes en Colombia desde la organización y las políticas. Universidad del Norte. Pensamiento \& gestión. (28):107-131.

Cobacho T., M.B. \& Bosch M., M. (2001). Contrastes de hipótesis en datos de panel. Universidad Politécnica de Cartagena. London School of Economics. 1-12

De la Vara G., J. L., Anes A., D., Sánchez D J (2004). Construcción de modelos de requisitos a partir de modelos de procesos y de metas. Departamento de Sistemas Informáticos y Computación. Universidad Politécnica de Valencia. 1-14.

Dini, M. y Stumpo, G. (2011). Políticas para la innovación en las pequeñas y medianas empresas en América Latina. Comisión Económica para América Latina y el Caribe (CEPAL). Chile Naciones Unidas. 1-165.

Estrada G., F. (2010). Herbert A. Simón y la economía organizacional. Universidad Externado de Colombia. Cuadernos de Economía. (46):169-198.

Estrada, S. y Dutrénit, G. (2007). Gestión del conocimiento en pymes y desempeño competitivo. Un análisis comparativo de Querétaro y Ciudad Juárez. Capítulo 13. Sistemas regionales de innovación: un espacio para el desarrollo de las Pymes. 272-298.

Etxebarria R., M. B. y Sánchez F., F. (2008). Diseño de un modelo de gestión basado en la pro actividad organizacional. Dpto.: Organización de Empresas (UPV/EHU). Revista de Dirección y Administración de Empresas. Número 15: 157-171.

Fúquene M., A. M., Ramírez M., D. C. y Castellanos D., O. F. (2009) Pronóstico para el fortalecimiento del desarrollo tecnológico. Revista ingeniería e investigación. 29 (3): (102-108).

Herrera U., M. F. y Osorio G., J. C. (2006). Modelo para la gestión de proveedores utilizando AHP difuso. Estudios Gerenciales. (99): 69-88.

Gil D, J.J. (2007). La gestión empresarial bajo el enfoque de las organizaciones inteligentes en la sociedad de la información. Fundación Miguel de Unamuno y Jugo. Negotium. 2 (6): 33-54.

Livacic R., C. (2009). Evolución de las Organizaciones: Un mundo en cambio. Universidad central. Revista de Estudios Politécnicos. 7 (12): 177-191.

Louffat O., E. (2003). Interconexión entre redes organizacionales, alianzas estratégicas y negociaciones. Un estudio multicaso. ESAN. Cuadernos de difusión 9 (16): 103-126.

Lloréns M, F. J. (1996). Una reflexión sobre la aplicación del análisis causal en el avance de la investigación del entorno organizacional. Cuadernos (30): 133-141.

Montaño H L (2000). La reapropiación internacional de modelos organizacionales. Algunas reflexiones sobre la experiencia japonesa. IZTAPALAPA. 54 (24): 245-264.

Mora J., J. (2006). Datos de panel en probit dinámicos. Estudios Gerenciales. 22 (101): 101-109.

Naclerio., A. (2010). - Sistemas productivos locales: políticas públicas y desarrollo económico / 1 a ed. - Buenos Aires: Programa Naciones Unidas para el Desarrollo - PNUD, 2010.

Orayen., J. (2008). Gestión del cambio empresarial en el contexto del sector Pyme manufacturero de la ciudad de Rosario Facultad de Ciencias Empresariales (Sede Rosario). Universidad Abierta Interamericana Secretaría de Investigación y Desarrollo. INFORME FINAL Proyecto de Investigación. 
Padilla H., S. 2008). Conocimiento tecnológico: el desafío para las PyMEs en México. Universidad Michoacana de San Nicolás de Hidalgo. Economía y Sociedad. 14 (22): 11-26.

Peña A., E. y Bastidas M., C. (2004). La pequeña y mediana empresa ante el fenómeno inmanente de la. Actualidad Contable. Mérida. Venezuela.FACES. 7(9): 52-60.

Pérez U., R- (2007). Estructura y cultura organizacional en la PyME colombiana: Análisis en Empresas Bogotanas. Universidad del Valle. Cuadernos de Administración. (38): 73-85.

Riquelme, A, Cravero, A, Saavedra, R (2009). Gestión del Conocimiento y Aprendizaje Organizacional: Modelo Adaptado para la Administración Pública Chilena. Universidad Autónoma de Chile: 43-61.

Rivas T., L. A. (1998). Nuevas formas de organización. Universidad ICESI. Estudios gerenciales. 14-45.

Simon, H. A. (1962) El comportamiento administrativo. Editorial Aguilar.

Urgal, G. B. (2007) ¿Cómo influye la infraestructura de producción en el rendimiento de las empresas manufactureras? Universidad de Vigo. Cuadernos de Gestión. 7(2): 13-27.

Yanes, E. V. (2001). La aproximación cognoscitiva en Administración de empresas. Principales aportaciones. Universidad de La Laguna. Investigaciones Europeas de Dirección y Economía de la Empresa. 7 (2): $123-138$ 